\title{
S-1 Monotherapy After Failure of Platinum Plus 5-Fluorouracil Chemotherapy in Recurrent or Metastatic Esophageal Carcinoma
}

\author{
TAKAHIKO ITO, YOSHITAKA HONMA, HIDEKAZU HIRANO, HIROKAZU SHOJI, \\ NATSUKO OKITA, SATORU IWASA, ATSUO TAKASHIMA, KEN KATO and NARIKAZU BOKU \\ Gastrointestinal Medical Oncology Division, National Cancer Center Hospital, Tokyo, Japan
}

\begin{abstract}
Background/Aim: Platinum plus 5-fluorouracil $(F P)$ is a first-line regimen of palliative chemotherapy for recurrent or metastatic esophageal squamous cell carcinoma (RM-ESCC). In this retrospective study, we evaluated the efficacy and safety of S-1 monotherapy as a salvage line treatment for RM-ESCC, focusing on the reasons for discontinuation of prior FP. Materials and Methods: The subjects of this study had RM-ESCC and received S-1 after failure of FP. Results: Eleven patients were enrolled. Nine patients were refractory and two were intolerant to prior $F P$. The median progression-free survival and overall survival time were 3.0 and 11.7 months, respectively. Overall response rate was $22.2 \%$ and disease control rate of the 11 patients was $36.4 \%$. Median relative dose intensity of 5-FU was $100 \%$ (range $=85-100 \%$ ). Conclusion: $S-1$ efficacy in RM-ESCC when given after FP was modest. Favorable OS may be attributed to good local control rather than to the efficacy of S-1 monotherapy.
\end{abstract}

Esophageal cancer was responsible for 11,543 deaths in Japan in 2013 (1). From the comprehensive registry of esophageal cancer in 2009, a total of 6,260 cases were registered by 276 institutions (2). Despite recent advances in the outcomes of many solid malignant neoplasms, the treatment of esophageal squamous cell carcinoma (ESCC) remains a difficult challenge, especially when it reaches an advanced stage.

For recurrent or metastatic ESCC (RM-ESCC), palliative chemotherapy consisting of platinum plus 5 -fluorouracil (FP)

Correspondence to: Yoshitaka Honma MD, Gastrointestinal Medical Oncology Division, National Cancer Center Hospital, 5-11 Tsukiji, Chuo-ku, 104-0045 Tokyo, Japan. Tel: +81 335422511, Fax: +81 335423815, e-mail: yohonma@ncc.go.jp

Key Words: Esophageal cancer, S-1, monotherapy, salvage, squamous cell carcinoma. is recognized as a standard first-line treatment (3). However, there is no standard second-line regimen after failure of FP. Paclitaxel and docetaxel have been widely used in daily practice based on the results of phase II trials $(4,5)$. After failure of taxane treatment, there is no effective salvage line treatment for RM-ESCC.

In Japan, S-1 is available for the treatment of esophageal cancer. S-1 is an oral fluoropyrimidine that contains tegafur, 5-chloro-2,4-dehydropyrimidine (gimeracil), and oteracil. Tegafur is a prodrug of 5-fluorouracil (5-FU) and gimeracil antagonizes dihydropyrimidine dehydrogenase, thereby inhibiting the degradation of 5-FU. In addition, oteracil inhibits the phosphorylation of 5-FU in the gastrointestinal tract, reducing its side-effects. If patients have already been treated with 5-FU and experienced progressive disease (PD), $\mathrm{S}-1$ is thought to be ineffective because it is a prodrug of 5-FU. Recently, it has been reported that S-1 monotherapy is efficacious after 5-FU failure in esophageal and gastric cancer, suggesting that the different mode of action of S-1 might have some efficacy even for patients resistant to 5-FU $(6,7)$. However, these previous reports did not explain whether FP was discontinued since patients were refractory or because they did not tolerate the treatment. Therefore, the purpose of this study was to clarify the safety and efficacy of S-1 monotherapy for RM-ESCC, focusing on the reason for discontinuation of prior FP.

\section{Patients and Methods}

Patient selection. This was a single center retrospective study. The subjects of this study were patients with RM-ESCC treated with S-1 monotherapy after failure of FP between August 2012 and August 2017 at National Cancer Center Hospital (NCCH). Patients who were treated with S-1 combined with radiotherapy or other cytotoxic agents were excluded. This retrospective study was approved by the Institutional Review Board of NCCH (2017-229).

Treatment. As the first line chemotherapy for advanced disease, standard FP comprised drip infusion of cisplatin $\left(80 \mathrm{mg} / \mathrm{m}^{2}\right)$ on day 
1 and continuous infusion of 5 -FU $\left(800 \mathrm{mg} / \mathrm{m}^{2} /\right.$ day $)$ on days 1 through 5 . For patients with renal dysfunction, nedaplatin at a dose of $90 \mathrm{mg} / \mathrm{m} 2$ was selected as an alternative to cisplatin. When FP was administered in combination with radiotherapy for treatment of local disease, a dose of $70 \mathrm{mg} / \mathrm{m}^{2}$ of cisplatin on day 1 and $700 \mathrm{mg} / \mathrm{m} 2 /$ day of 5 -FU by continuous infusion on days 1 through 4 was used. In some cases, triplet chemotherapy in which cisplatin and docetaxel were administered at a dose of $70 \mathrm{mg} / \mathrm{m}^{2}$ of docetaxel on day 1 , and $750 \mathrm{mg} / \mathrm{m}^{2}$ of 5 -FU by continuous infusion on days 1 through 5 (DCF regimen) was used.

With regard to S-1, patients generally received two oral doses of the drug at a dose of $40 \mathrm{mg} / \mathrm{m}^{2}$, twice daily, for 4 weeks, followed by 2 weeks rest; this was repeated every 6 weeks. The actual dose of $\mathrm{S}-1$ at each administration was calculated according to the patient's body surface area (BSA): $40 \mathrm{mg}$ for BSA $<1.25 \mathrm{~m}^{2}, 50 \mathrm{mg}$ for $1.25 \mathrm{~m}^{2} \leq \mathrm{BSA}<1.5 \mathrm{~m}^{2}$, and $60 \mathrm{mg}$ for $1.5 \mathrm{~m}^{2} \leq \mathrm{BSA}$. $\mathrm{S}-1$ monotherapy was repeated until disease progression or the occurrence of severe adverse events.

Data collection. The following information was retrospectively collected from the medical records and radiological images: age, gender, performance status, clinical stage (according to the 7th Edition of the UICC TNM Classification of Malignant Tumors), prior treatments, relative dose intensity (RDI) of 5-FU in prior chemotherapy, reason for discontinuation of prior FP (patients were refractory or intolerant to treatment), status of local control, and treatment after S-1 monotherapy.

Evaluation. Tumor response was evaluated according to the Response Evaluation Criteria in Solid Tumors guidelines version 1.1. Overall response rate (ORR) is defined as the proportion of patients who had a partial or complete response. In our study, disease control rate (DCR) was defined as the percentage of patients who had achieved complete response, partial response or stable disease in patients with target lesions (TLs) and complete response or disease stabilization in patients without TLs. Toxicity was assessed by an attending doctor and evaluated according to the Common Terminology Criteria for Adverse Events version 4.0 (CTCAE v4.0). Overall survival (OS) was calculated from the day of initiating S-1 monotherapy to the time of death from any cause, or censored at the last follow-up. Progression-free survival (PFS) was calculated for the period from the first day of S-1 monotherapy to the time of first progression documentation or death from any cause, or censored at the last confirmation of follow-up without progression or death.

Statistical analysis. Survival curves were drawn with the KaplanMeier method and compared using the log-rank test. All statistical analyses were performed using Statistical Package for Social Sciences (SPSS) software (version 25; SPSS, Inc, Chicago, IL, USA).

\section{Results}

Patient characteristics. Between August 2012 and August 2017, 42 patients with RM-ESCC refractory or intolerant to FP were treated with S-1. Among these, 23 patients were excluded as they had been treated with a combination of cytotoxic agents other than FP or radiotherapy. Eight of the remaining 19 patients were excluded because they received S-1 as maintenance therapy after FP to which they were not

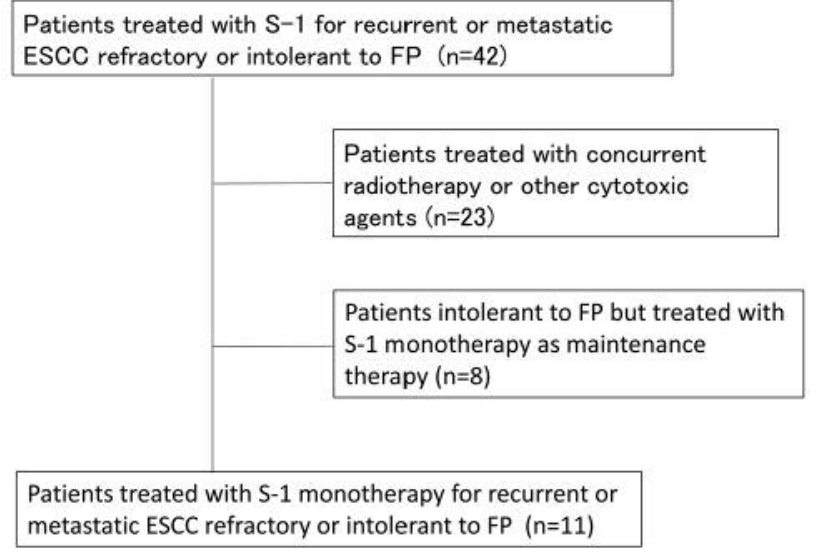

Figure 1. Flowchart of patient selection.

either refractory or intolerant. The remaining 11 patients were identified as the subjects of this study (Figure 1).

All patients were treated with FP as first line treatment; 9 patients received 5-FU plus cisplatin and 2 patients received 5-FU plus nedaplatin. Of these, 3 patients received DCF regimen as induction chemotherapy before FP. Nine of 11 patients discontinued FP regimen due to disease progression (refractory group), and 2 patients due to intolerance (intolerant group). The reasons for intolerance were grade 2 fatigue in one patient and grade 3 anorexia in the other.

Patient characteristics at the initiation of $\mathrm{S}-1$ are summarized in Table I. Most cases (10 of 11 patients) were male, and the median age was 69 years. Eastern Cooperative Oncology Group performance status (ECOG PS) was 0 in 5 patients, and 1 in 6 patients. Four patients had initially unresectable disease and 7 had recurrence. Prior treatment in nine of 11 patients produced good local control (defined as no residual tumor or recurrence at the primary site following endoscopy and CT imaging). Reduction of the initial S-1 dose was required for 4 patients due to renal dysfunction.

Outcomes. Median follow-up period was 11.8 months (range=2.7-27.7 months). Median PFS and median survival time (MST) were 3.0 months [95\% confidence interval $(C I)=0.303-5.697]$ and 11.7 months $(95 \% \mathrm{CI}=8.36-15.04)$, respectively (Figure 2). MST in the refractory group was 11.7 months $(95 \% \mathrm{CI}=0.97-22.4)$ and that of the intolerant group was 9.5 months [95\% CI=not assessed (N.A.)] $(p=0.204)$ (Figure 3). Median PFS in the refractory group was 2.7 months $(95 \% \mathrm{CI}=1.82-3.58)$ and that in the intolerant group was 3.0 months $(95 \% \mathrm{CI}=\mathrm{N} . \mathrm{A}).(p=0.621)$ (Figure 3$)$.

Therapeutic effect. Among 9 patients with TLs, 2 achieved partial response (PR); one in the refractory group and the other in intolerant groups, while the remaining 7 patients 
Table I. Therapeutic courses of patients.

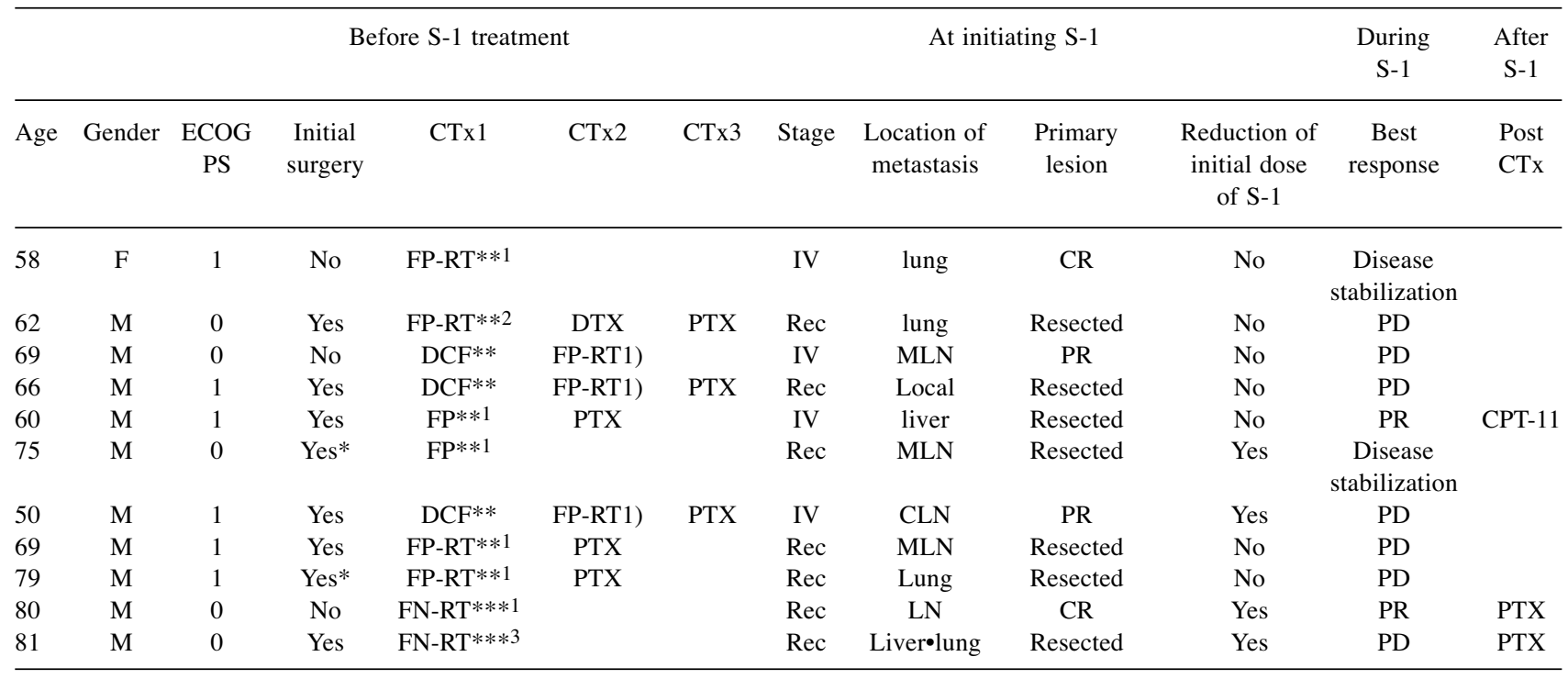

FP: 5-FU+Cisplatin FP-RT: 5-FU+Cisplatin+RT; FN-RT: 5-FU+Nedaplatin+RT; DTX: docetaxel; PTX: paclitaxel; DCF: docetaxel+cisplatin+5-FU; Surg.: surgery; Rec.: recurrence; MLN: mediastinal lymph node; CLN: cervical lymph node; *endoscopic resection; **intolerance; ***refractory. ${ }^{1}$ RDI: $100 \%$; ${ }^{2}$ RDI: $95.6 \%$; ${ }^{3}$ RDI: 85.0\%. Best overall response (target lesion, N=9): CR 0 (0\%), PR 2 (22.2\%), SD 0 (0\%), PD 7 (77.7\%); (nontarget lesion, $\mathrm{N}=2)$ : CR $0(0 \%)$, disease stabilization $2(100 \%)$, PD $0(0 \%)$.

showed progressive disease $(\mathrm{ORR}=22 \%)$ (Table I). The other 2 patients without TLs achieved disease stabilization, resulting in a $36.4 \%$ DCR of 11 patients (4/11). The median RDI of $5-\mathrm{FU}$ was $100 \%$, (range $=85-100 \%$ ). Median RDI of 5-FU in PD patients was $100 \%$ (range $=85-100 \%$ ) and $100 \%$ (all 100\%) in non-PD patients.

Toxicity of S-1 monotherapy. The most common S-1 related adverse event was grade 1 oral mucositis $(36.4 \%)$. The other toxicities were grade 2 constipation $(9.1 \%)$, grade 2 general fatigue $(9.1 \%)$, grade 1 anorexia (18.2\%) and grade 1 nausea (18.2\%). Although grade 3 leukopenia was seen in one patient $(9.1 \%)$, there were no serious adverse events or treatment-related deaths.

Treatment after S-1 monotherapy. With regard to subsequent therapy after S-1 treatment, only 1 patient in the refractory group received irinotecan monotherapy, while 8 received best supportive care. On the other hand, both patients in the intolerant group received paclitaxel after S-1 monotherapy. Clinical courses of all the patients are summarized in Table I.

\section{Discussion}

In Asian regions, the majority of head and neck cancers are squamous cell carcinomas such as esophageal carcinoma. There are some treatment options for these malignancies, such as fluoropyrimidine, platinum, taxanes, cetuximab (antiepidermal growth factor receptor antibody) and nivolumab (anti PD-1 antibody). By contrast, there are only a few agents available for treatment of RM-ESCC; therefore, S-1 is used for patients who have had prior FP treatment. Nomura et al. reported the efficacy of salvage line chemotherapy for RMESCC (8). In this study, patients with RM-ESCC who were refractory to (or intolerant of) the three agents (platinum, taxane, and fluorouracil) were retrospectively reviewed. Of these, 16 patients received fluorouracil (14 of 16 received S1 monotherapy) as a salvage chemotherapy. The ORR was $13.3 \%$, DCR was $66.7 \%$ and MST was 12.9 months, respectively (8), but the number of patients who were intolerant was not mentioned. In addition, Akutsu et al. reported the efficacy of S-1 monotherapy as a second- or third-line regimen. The study monitored twenty patients who received S-1 monotherapy after their tumors became refractory to FP, or FP plus radiotherapy or docetaxel. They reported that the ORR, DCR and MST were 25\%, 60\% and 330 days, respectively (6). However, similar to the Nomura study mentioned above, the percentage of patients who could not tolerate FP was not reported. In our study, the ORR was $22.2 \%$ and OS was 11.7 months; these values are similar to those of previous reports. Together these results indicate that S-1 exhibits modest activity for RM-ESCC treatment, even after failure of FP. As for the re-challenge of taxanes, Iizumi et al. reported that paclitaxel was ineffective in gastric cancer 

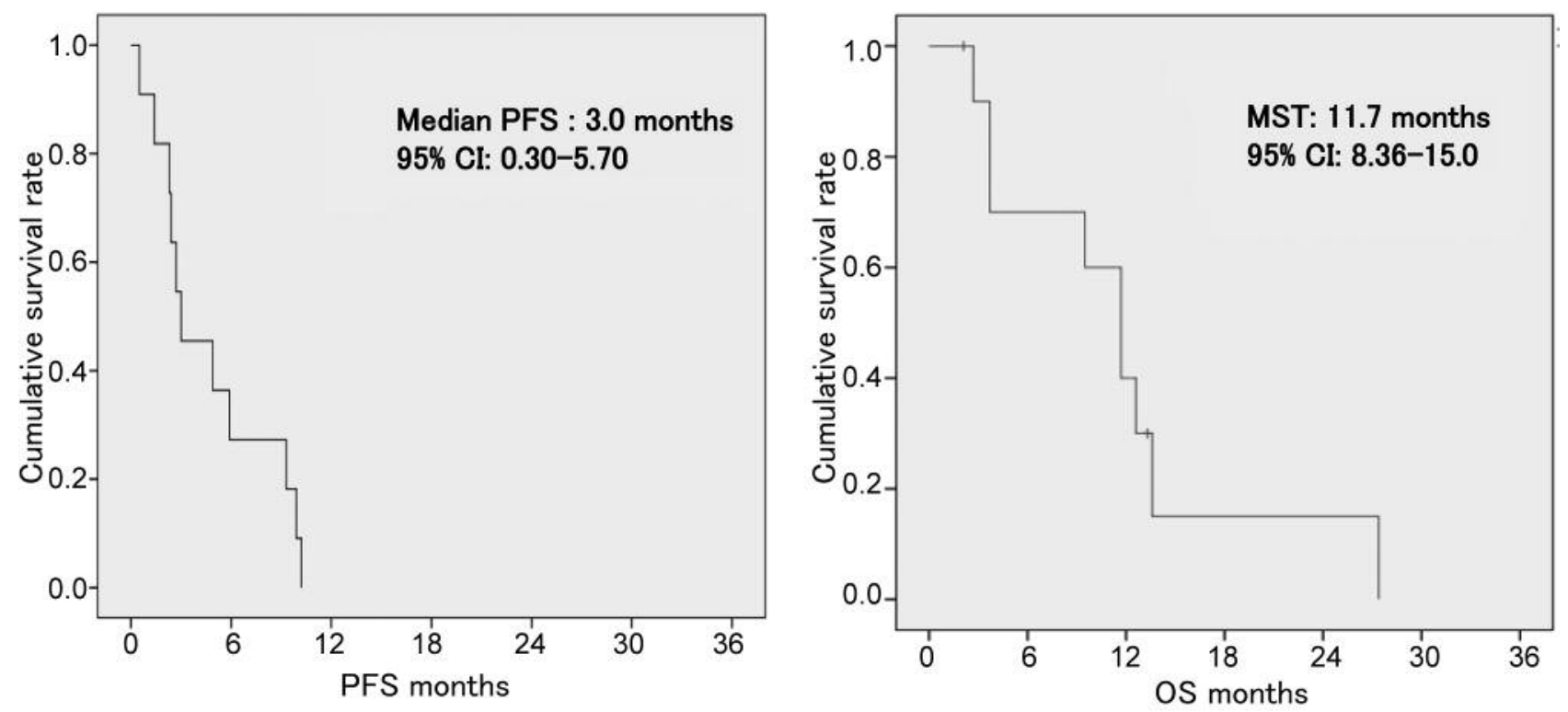

Figure 2. median PFS and OS.
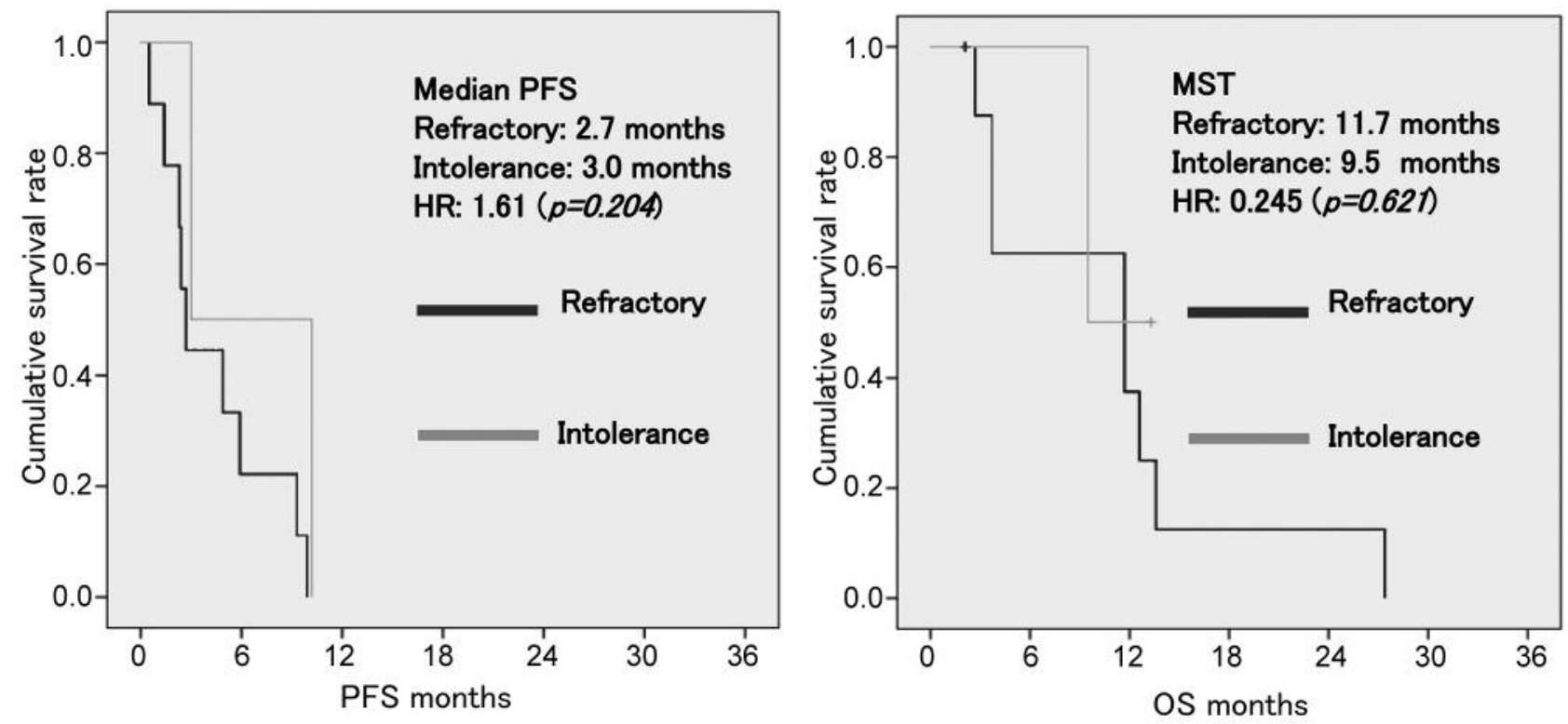

Figure 3. Median PFS and OS in the refractory and intolerant group.

patients who received prior treatment with 5-FU, CDDP and docetaxel (9). It is well known that paclitaxel and docetaxel elicit the same biological program that leads to resistance. It is still unclear why S-1 monotherapy is effective for some patients even after prior FP treatment has failed. However, the different mode of action of S-1 in tumor cells, such as DPD inhibition, might contribute to its efficacy. We note that, despite the positive effects of S-1 treatment, more than half of the patients showed progressive disease and the disease control rate was relatively low, at $36.4 \%$.

We hypothesized that S-1 monotherapy would show efficacy in patients with insufficient RDI for prior 5-FU. 
Hence, we examined the RDI of each patient, and found that the median RDI of prior 5-FU in 11 cases was $100 \%$ (range $=85-100 \%$ ). Although the sample size was quite small, we did not detect a relationship between the RDI of prior $5-\mathrm{FU}$ and the efficacy of S-1 monotherapy. It is still unknown why S-1 monotherapy is efficacious in some patients after failure of FP. Therefore, further work is required in order to determine the molecular mechanism that underlies efficacy in this context. This will help provide a better patient selection strategy.

Previous phase II trials of FP as a first line regimen reported 3.6-6.2 months for PFS and 5.5-7.6 months for OS, meaning that post-treatment survival after FP failure was approximately 1.0 to 1.9 months (10-12). Moreover, the MST of ESCC treated with docetaxel as a second line regimen after FP failure was reported as 3.3-4.3 months or 5.4 months, depending on the study $(8,13)$. In our study, in spite of the low DCR and short PFS, the MST was significantly longer, at approximately 10 months. As this was a retrospective study with a small number of patients, there may have been some selection bias. For example, most patients had experienced good local control due to their prior treatments. It is well known that primary tumors of the esophagus cause complications such as obstruction, fistula and bleeding. Malignant esophageal stenosis has been reported as an independent prognostic factor in ESCC (14), showing that patients with good local control were able to maintain a better oral intake and general condition. In this study, the primary tumor was already well controlled in many patients at the time when S-1 treatment was initiated. We infer that the favorable OS we observed might be due to control of the primary tumor, rather than being a result of S-1 efficacy.

In this study, many of the subjects had received taxane before initiating $S-1$. Recently, immune checkpoint inhibitors such as anti-PD-1/PD-L1 antibody have provided a new treatment opportunity for various cancers. A recent phase II trial has shown that Nivolumab, a fully human IgG4 monoclonal antibody inhibitor of programmed death-1 (PD1) showed promising activity for patients whose RM-ESCC was refractory to fluoropyrimidine, platinum, and taxane, or who were intolerant to these drugs (15). According to this trial, the MST and centrally assessed PFS were 10.8 months (95\% CI=7.4-13.3) and 1.5 months (95\%CI=1.4-2.8), respectively. ORR and DCR were $25 \%$ and $67 \%$, respectively. Based on these results, Nivolumab has the potential to become the new first-choice for salvage line treatment after FP for RM-ESCC. Other reports suggest that concomitant exposure to immune checkpoint inhibitors enhances the efficacy of chemotherapy in malignancies such as non-small cell lung cancer and head and neck squamous cell carcinoma $(16,17)$. Thus, treatment of RM-ESCC may be more effective if chemotherapy follows an initial course of Nivolumab. Since the combination of taxane with platinum leads to cumulative neurotoxicity, it is sometimes difficult to use them in clinical practice. Therefore, S-1 monotherapy might be an alternative to taxane in patients who have previously been treated with FP and immunotherapy.

In conclusion, the response rate of S-1 monotherapy was modest $(22 \%)$ and the DCR was low (36.4\%) in cases after FP failure, resulting in a PFS of approximately 3 months. Favorable OS may be attributed to good local control by the prior treatment, rather than the effectiveness of S-1 monotherapy. Further studies exploring the optimal salvage line treatment for RM-ESCC are warranted.

\section{Conflicts of Interest}

The Authors have no conflict of interest in regard to this study.

\section{Authors' Contributions}

TI and $\mathrm{YH}$ designed the study, performed data collection and analyzed the data. TI, YH and NB interpreted the results and drafted the manuscript. All Authors contributed to the treatment and gave approval of the final version of submission.

\section{References}

1 Vital Statistics Japan (2013). Ministry of Health, Labour and Welfare. Available from: https://www.mhlw.go.jp/english/ database/db-hw/index.html

2 Tachimori Y, Ozawa S, Numasaki H, Fujishiro M, Matsubara H, Oyama $\mathrm{T}$, Shinoda $\mathrm{M}$, Toh $\mathrm{Y}$, Udagawa $\mathrm{H}$ and Uno $\mathrm{T}$ : Comprehensive registry of esophageal cancer in Japan, 2009. Esophagus 13: 110-137, 2016. PMID: 27110229. DOI: 10.1007/ s10388-016-0531-y

3 Cooper JS, Guo MD, Herskovic A, Macdonald JS, Martenson JA Jr., Al-Sarraf M, Byhardt R, Russell AH, Beitler JJ, Spencer S, Asbell SO, Graham MV and Leichman LL: Chemoradiotherapy of locally advanced esophageal cancer: long term follow-up of a prospective randomized trial (RTOG 85-01). Radiation Therapy Oncology Group. JAMA 281: 1623-1627, 1999. PMID: 10235156.

4 Ilson DH, Ajani J, Bhalla K, Forastiere A, Huang Y, Patel P, Martin L, Donegan J, Pazdur R, Reed C and Kelsen DP: Phase II trial of paclitaxel, fluorouracil, and cisplatin in patients with advanced carcinoma of the esophagus. J Clin Oncol 16: 18261834, 1998. PMID: 9586897. DOI: 10.1200/JCO.1998.16.5.1826

5 Ajani JA, Ilson DH, Daugherty K, Pazdur R, Lynch PM and Kelsen DP: Activity of taxol in patients with squamous cell carcinoma and adenocarcinoma of the esophagus. J Natl Cancer Inst 86: 10861091, 1994. PMID: 791276. DOI: 10.1093/jnci/ 86.14.1086

6 Akutsu Y, Kono T, Uesato M, Hoshino I, Narushima K, Hanaoka T, Tochigi T, Semba Y, Qin W and Matsubara H: S-1 monotherapy as second- or third line chemotherapy for unresectable and recurrent esophageal squamous cell carcinoma. Oncology 84: 305310, 2013. PMID: 23595163. DOI: 10.1159/000348294

7 Lv F, Liu X, Wang B, Guo H, Li J, Shen L and Jin M: S-1 monotherapy as second line chemotherapy in advanced gastric cancer patients previously treated with cisplatin/infusional fluorouracil. Int J Clin Exp Pathol 7: 3293-3298, 2014. PMID: 25031751. 
8 Nomura M, Iwasa S, Tsushima T, Kato K, Yasui H, Boku N, Muto $\mathrm{M}$ and Muro K: Active salvage chemotherapy versus best supportive care for patients with recurrent or metastatic squamous cell carcinoma of the esophagus refractory to fluorouracil, platinum, and taxane. Cancer Chemother Pharmacol 78(6): 1209-1216, 2016. PMID: 27778070. DOI: 10.1007/ s00280-016-3179-3

9 Iizumi S, Takashima A, Narita Y, Tajika M, Muro K, Kawai S, Yasui H, Matsushima T, Takahari D, Nagashima K and Boku N: Efficacy and safety of taxane monotherapy in advanced gastric cancer refractory to triplet chemotherapy with docetaxel, cisplatin, and S-1: a multicenter retrospective study. Cancer Chemother Pharmacol 80: 575-582, 2017. PMID: 28730292. DOI: $10.1007 / \mathrm{s} 00280-017-3397-3$

10 Bleiberg H, Conroy T, Paillot B, Lacave AJ, Blijham G, Jacob JH, Bedenne L, Namer M, De Besi P, Gay F, Collette L and Sahmoud T: Randomized phase II study of cisplatin and 5-FU versus cisplatin alone in advanced squamous cell esophageal cancer. Eur J Cancer 33: 1216-1220, 1997. PMID: 9301445.

11 Hayashi K, Ando N, Watanabe H, Ide H, Nagai K, Aoyama N, Takiyama $\mathrm{W}$, Ishida $\mathrm{K}$, Isono $\mathrm{K}$, Makuuchi $\mathrm{H}$, Imamura $\mathrm{M}$, Shinoda M, Ikeuchi S, Kabuto T, Yamana H and Fukuda H: Phase II evaluation of protracted infusion of cisplatin and 5fluorouracil in advanced squamous cell carcinoma of the esophagus: a Japan Esophageal Oncology Group (JEOG) trial (JCOG9407). Jpn J Clin Oncol 31: 419-423, 2001. PMID: 11689594. DOI: $10.1093 / \mathrm{jjco} / \mathrm{hye} 090$

12 Lorenzen S, Schuster T, Porschen R, Al-Batran SE, Hofheinz R, Thuss-Patience P, Moehler M, Grabowski P, Arnold D, Greten T, Müller L, Röthling N, Peschel C, Langer R and Lordick F: Cetuximab plus cisplatin-5-fluorouracil versus cisplatin-5fluorouracil alone in first-line metastatic squamous cell carcinoma of the esophagus: a randomized phase II study of the Arbeitsgameinschaft Internistische Onkologie. Ann Oncol 20: 1667-1673, 2009. PMID: 19549707. DOI: 10.1093/annonc/ mdp069
13 Moriwaki T, Kajiwara T, Matsumoto T, Suzuki H, Hiroshima Y, Matsuda K, Hirai S, Yamamoto Y, Yamada T, Sugaya A, Kobayashi M, Endo S, Ishige $\mathrm{K}$, Nishina $\mathrm{T}$ and Hyodo I: Survival analysis of platinum-refractory patients with advanced esophageal cancer treated with docetaxel or best supportive care alone: a retrospective study. Dis Esophagus 27: 737-743, 2014. PMID: 24917486. DOI: 10.1111/dote. 12246

14 Yang YS, Hu WP, Ni PZ, Wang WP, Yuan Y and Chen LQ: Esophageal luminal stenosis is an independent prognostic factor in esophageal squamous cell carcinoma. Oncotarget 27: 43397-43405, 2017. PMID: 28118615. DOI: 10.18632/ oncotarget.14762

15 Kudo T, Hamamoto Y, Kato K, Ura T, Kojima T, Tsushima T, Hironaka S, Hara H, Satoh T, Iwasa S, Muro K, Yasui H, Minashi K, Yamaguchi K, Ohtsu A, Doki Y and Kitagawa Y: Nivolumab treatment for oesophageal squamous-cell carcinoma: an open label, multicentre, phase 2 trial. Lancet Oncol 18: 631-639, 2017. PMID: 28314688. DOI: 10.1016/ S1470-2045(17)30181-X

16 Dwary AD, Master S, Patel A, Cole C, Mansour R, Mills G, Koshy N, Peddi P, Burton G, Hammoud D and Beedupalli K: Excellent response to chemotherapy post immunotherapy. Oncotarget 8: 91795-91802, 2017. PMID: 29207685. DOI: 10.18632/oncotarget.20030

17 Schvartsman G, Peng SA, Bis G, Lee JJ, Benveniste MFK, Zhang J, Roarty EB, Lacerda L, Swisher S, Heymach JV, Fossella FV and William WN: Response rates to single-agent chemotherapy after exposure to immune checkpoint inhibitors in advanced non-small cell lung cancer. Lung Cancer 112: 90-95, 2017. PMID: 29191606. DOI: 10.1016/j.lungcan.2017.07.034

Received May 2, 2019

Revised May 29, 2019

Accepted May 30, 2019 